\title{
Milli-arcsecond binaries
}

\author{
R. M. Torres ${ }^{1}$, L. Loinard ${ }^{1}$, A. J. Mioduszewski ${ }^{2}$ and L. F. Rodríguez ${ }^{1}$ \\ ${ }^{1}$ Centro de Radiastronomía y Astrofísica, Universidad Nacional Autónoma de México \\ Apartado Postal 72-3 (Xangari), 58089 Morelia, Michoacán, México \\ email: $r$.torres, 1 . loinard, 1.rodriguez@astrosmo. unam.mx \\ ${ }^{2}$ National Radio Astronomy Observatory, Array Operations Center \\ 1003 Lopezville Road, Socorro, NM 87801, USA \\ email: amiodusz@aoc.nrao.edu
}

\begin{abstract}
As part of an astrometric program (see Loinard et al. in this volume), we have obtained an unprecedented sample of high-resolution $(\sim 1$ mas) VLBA images of several nearby young stellar systems. As was to be expected, these images revealed interesting new characteristics of these objects. Perhaps the most interesting of these characteristics is the detection of a high rate of very tight binary stars (separations of a few mas) in our sample: of the 9 objects observed to date, 5 are binaries of this type.
\end{abstract}

Keywords. binaries: general, stars: individual (T Tauri Stars), radiation mechanisms: nonthermal, astrometry

\section{Introduction}

Multiple studies have led to the well-known conclusion that main-sequence stars in the Solar neighborhood show a high multiplicity rate: $\sim 55 \%$ for solar-type stars (Duquennoy \& Mayor 1991), and 35 to 42\% for M-dwarfs (Reid \& Gizis 1997; Fischer \& Marcy 1992). Young stars also exhibit a high multiplicity rate: $\sim 50 \%$ with separations of between 0.02 and 1 arcseconds (Köhler \& Leinert 1997; Duchêne et al. 2004; Konopacky et al. 2007). Indeed, several star-forming regions appear to show multiplicity rates even higher than those of main sequence stars (Prosser et al. 1994; Duchêne et al. 1999; Bouvier et al. 1997). This shows that the multiplicity must be already established in the very early phases of star-formation.

\section{Observations}

We have chosen from the literature a list of 9 young stellar objects associated with nonthermal radio emission: 5 in Taurus, and 4 in the Ophiuchus complex. Those sources are low-mass stars (with the exception of $\mathrm{S} 1$ which is a main sequence B star). We make use of a series of six continuum $3.6 \mathrm{~cm}(8.42 \mathrm{GHz})$ observations of each source obtained roughly every two or three months. Our calibrators are very compact extragalactic sources whose absolute positions are know to better than about 1 milli-arcsecond. The data were edited and calibrated in a standard fashion using the Astronomical Image Processing System (AIPS).

\section{Results and discussion}

In this work we find that $\sim 70 \%$ of the objects in our sample are multiple stellar systems with separations of a few mas. In the Ophiuchus complex we found a new member in the each of the stellar systems S1, DoAr 21 and VSSG 14, and two new members in WL 

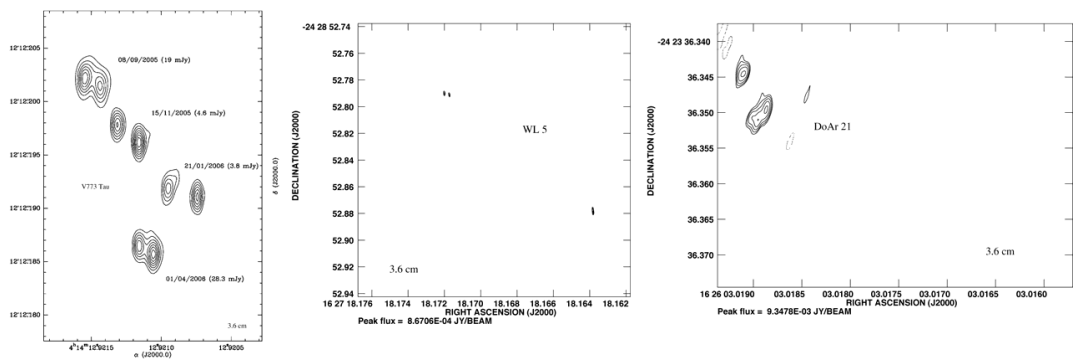

Figure 1. Multiple stellar systems. The first 4 epochs for V773 Tau in Taurus; and the first epoch for WL 5 and DoAr 21 in $\rho$-Ophiuchus.

5 (see Fig. 1). In addition, we find extended structure around several systems. In the case of Taurus, we knew that $\mathrm{T}$ Tau Sb was a member of a triple system, but among these three members we only detect one with a non-thermal radio emission. V773 Tau was previously known to be a quadruple system, and we detect 2 out of the 4 members. Finally, Hubble 4 and HDE 283572, as well as HP Tau/G2 are apparently single.

It is quite unlikely that $70 \%$ of all young stellar systems are tight binaries such as those found in our VLBA observations. The high binary rate found here is more likely the result of selection effects. The systems considered here were selected because they were known to be non-thermal emitters, so the high binary rate may indicate that tight binaries are more likely to emit non-thermal radio emission than looser binaries or single stars. This idea is reinforced by the observations of V773 Tau (Fig. 1) where we find that the emitted flux is a clear function of the separation between the two stars (3.8 mJy when they are most separated against $28.3 \mathrm{mJy}$ when they are closest). While the physical mechanism leading to this behavior is not clear, this result certainly suggests a relation between the radio flux and separation. Also, the fraction of tight binaries appears to be larger in $\rho$-Oph than in Taurus.

\section{Perspectives}

For pre-main sequence stars, no reliable empirical mass determination is available. For the binary stars detected here, a direct mass determination will be possible using Kepler's third law and the measured relative motions. We are currently obtaining new observations for these multiple sources. New observations will cover complete orbits of the sources and will allow the determination of their orbital parameters and masses.

\section{References}

Bouvier, J., Rigaut, F., \& Nadeau, D. 1997, A\&A, 323, 139

Duchêne, G., Bouvier, J., \& Simon, T. 1999, A\& A, 343, 831

Duchêne, G., Bouvier, J., Bontemps, S., Andr, P., \& Motte, F. 2004, A\&A, 427, 651

Duquennoy, A. \& Mayor, M. 1991, A\&A, 248, 485

Fischer, D. A. \& Marcy, G. W. 1992 ApJ, 396, 178

Köhler, R. \& Leinert, C. 1998 A\&A, 331, 977

Konopacky, Q. M., Ghez, A. M., Rice, E. L., \& Duchêne, G. 2007 ApJ, 663, 394

Prosser, C. F., Stauffer, J. R., Hartmann, L., Soderblom, D. R., Jones, B. F., Werner, M. W., \& McCaughrean, M. J. 1994 ApJ, 421, 517

Reid, I. N. \& Gizis, J. E. 1997 AJ, 113, 2246 